\title{
Preliminary investigations of Agrobacterium-mediated transformation in indica rice MR219 embryogenic callus using gusA gene
}

\author{
Zuraida Ab. Rahman', Zulkifli Ahmad Seman', Naziah Basirun', Advina Lizah Julkifle², \\ Zamri Zainal $^{3}$ and Sreeramanan Subramaniam ${ }^{2}$
${ }^{1}$ Biotechnology Research Centre, MARDI Headquarter, Persiaran Mardi-UPM, 43400 Serdang, Selangor, Malaysia. ${ }^{2}$ School of Biological Sciences, Universiti Sains Malaysia (USM), Georgetown, 11800, Penang, Malaysia.
${ }^{3}$ Faculty of Science and Technology, Universiti Kebangsaan Malaysia, 43600 Bangi, Selangor, Malaysia.

Accepted 17 June, 2011

\begin{abstract}
Preliminary steps in the genetic transformation of indica rice MR219 was investigated in the plantAgrobacterium tumefaciens interaction. Agrobacterium tumefaciens strain LBA 4404 carrying a binary vector pCAMBIA 1305.2 harboring the modified GUS gene driven by the CaMV 35S promoter was used. Various transformation parameters influences were optimized using embryogenic calli via $\beta$ glucuronidase (GUS) as a reporter marker. Various transformation parameters were optimized including bacterial concentration, age of embryogenic callus, pre-culture period, wounding technique, cocultivation period, immersion time and dry time before co-cultivation, acetosyringone (AS) concentration, $\mathrm{pH}$ of co-cultivation media and temperature of the co-cultivation period. The expression of the transient gusA gene in the plant genome was preliminary confirmed by histochemical GUS assay activity (as blue spots). The results from transient gusA gene expression of calli suggested that the Agrobacterium-mediated transfer system of T-DNA in indica rice MR219 was highly efficient. Therefore, the investigation of factors that influence T-DNA delivery is an important first step in the utilization of Agrobacterium in the transformation of indica rice MR219 calli.
\end{abstract}

Key words: Indica rice MR219, Agrobacterium tumefaciens, GUS expression.

\section{INTRODUCTION}

Oryza sativa L., indica-type rice, is an indubitably important staple food for many Asian regions. In Malaysia, indica rice variety MR219 was developed by Malaysian Argicultural Research and Development Institute (MARDI) to boost rice yield up to $10 \mathrm{mt} / \mathrm{ha} ; 10$ to $15 \%$ higher than the previous MR84 variety. MR219 potential to produce high yields depends on the grain sizes which weigh 28 to $30 \mathrm{mg}$ and the number of grain per panicle which can reach 200. In addition, MR219 is resistant to bacterial leaf blight and blast disease with short maturation period from 105 to 111 days (http://www.agnet.org/library/rh/2002010a/).

Nevertheless, further researches to improve the quality

\footnotetext{
${ }^{*}$ Corresponding author. E-mail: azuraida@mardi.gov.my.
}

of MR219 are essential. Jiao et al. (2010) and Miura et al. (2010) recently showed that low number of tiller increaseed productivity with the increase of number of panicles and number of grains per panicles which is regulated by the over expression of OSSPL14 gene.

Genetic transformation has become an important means in the crop improvement strategies. The system is gene specific and less laborious with less time consumption as compared to the traditional rice breeding practice. Agrobacterium tumefaciens has been reported to be an excellent tool in various successful production of genetically modified (GM) crops and ornamentals including barley (Shrawat et al., 2007), banana (Sreeramanan et al., 2006), dragon fruit (Kavitha et al., 2010), cassava (Bull et al., 2009), japonica and indica rice cultivar Kasalath (Kumar et al., 2005; Nishimura et al., 2006; Hiei and Komari, 2008), and orchid 


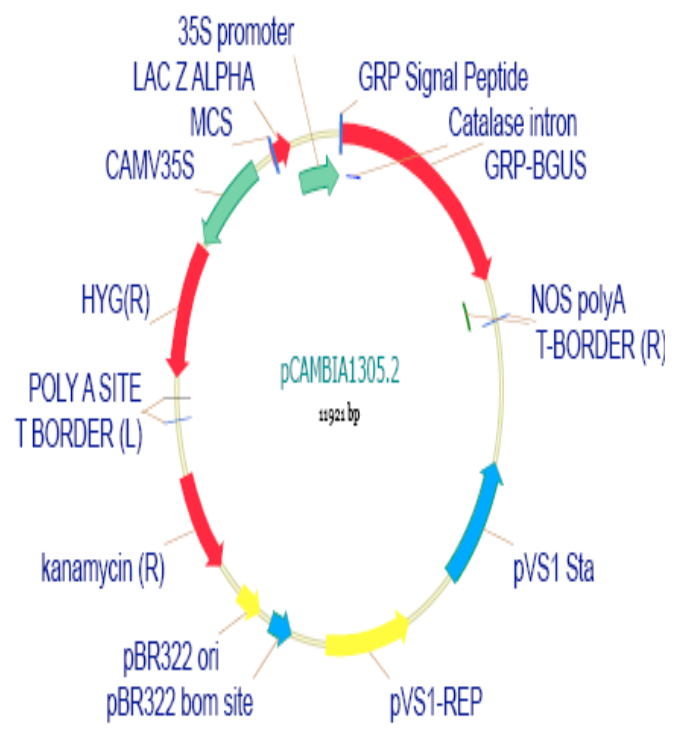

Figure 1. Schematic diagram of the plasmid PCAMBIA 1305.2. The binary vector pCAMBIA1304 (CSIRO, Australia) harboring the reporter gusA and mgfp5 genes driven by the CaMV 35S promoter.

(Sreeramanan and Zuraida, 2010; Sreeramanan and Xavier, 2010). A. tumefaciens is a favourable tool of genetic transfer in rice transformation (Nishimura et al., 2006; Hiei and Komari, 2008) since it allows stable, single-copy sequences to be inserted into the rice plant genomes efficiently (Kumar et al., 2005; Nishimura et al., 2006; Hiei and Komari, 2008). Early detection of plant transformation events is necessary for the optimization of gene transfer into the rice genome. Reporter gene such as Escherichia coli gusA (uidA) gene encoding for enzyme $\beta$-glucuronidase (GUS) is used to provide a clear indication of the expression, transient or stable, of transferred genes in plant transgenic cells (Jefferson , 1987; Hiei et al., 1997; Sreeramanan et al., 2006; Xing et al., 2007; Sreeramanan and Xavier, 2010).

Various interactions and progress on different host plant for transformation require continuous investigation. Hui et al. (2004) reported that in vitro response is highly genotype dependent, especially in indica rice, which was difficult to have a uniform Agrobacterium-mediated transformation system. Hiei et al. (1997) reviewed various key factors required in Agrobacterium-mediated transfer of genes into rice such as vir gene induction, active cell division in the target tissue ( $\mathrm{s}$ ), medium composition, genotype and Agrobacterium strains and vectors. In the study, several parameters considered crucial for the transformation efficiency of MR219 rice variety using Agrobacterium-mediated system were assessed by using GUS as a reporter gene. There was no system report of Agrobacterium-mediated transformation carried out on this rice MR 219 variety until now.

\section{MATERIALS AND METHODS}

Embryogenic calli used in this study were derived from mature rice seeds (Zuraida et al., 2007). The calli were maintained on agar solidified embryogenic media containing MS including B5 vitamin (Murashige and Skoog, 1962) and supplemented with $1 \mathrm{mg} / \mathrm{L} \mathrm{2,4-}$ $\mathrm{D}, 10 \mathrm{mg} / \mathrm{L} \mathrm{NAA}, 30 \mathrm{~g} / \mathrm{L}$ sucrose and $3.5 \mathrm{mg} / \mathrm{L}$ agar (Zuraida et al., 2007). The medium was adjusted to $\mathrm{pH} 5.7$ with $\mathrm{KOH}$ prior to autoclaving. 3 to 6 weeks-old embryogenic callus was used for the transformation of indica rice MR219.

\section{Agrobacterium strain and plasmid}

A. tumefaciens strain LBA4404 harbouring the plasmid pCAMBIA1305.2 (http://www.cambia.org.au/) with the $\beta$ glucuronidase (GUS) gene (uidA version GUSPlus ${ }^{\mathrm{TM}}$ and the $h p t$ gene) interrupted with a plant intron (GUS-INT) driven by the Cauliflower Mosaic Virus 35S (CaMV 35S) and nopaline synthase terminator as selectable marker gene was used for the transformation (Figure 1). This vector has hygromycin phosphotransferase (hptll) gene in T-DNA region driven by CaMV35S promoter and CaMV35S polyA terminator, which confers resistance to the antibiotic hygromycin as a plant selection marker. Neomycin phosphotransferase (nptll) gene is located outside the T-DNA region driven by the CaMV35S. Nopaline synthase (NOS) terminator confers resistance to the antibiotic kanamycin as a bacterial selection marker.

\section{Inoculation and co-cultivation with A. tumefaciens}

Single colony of Agrobacterium from freshly subcultured plate was grown overnight in Luria-Bertani (LB) liquid medium containing 50 $\mathrm{mg} / \mathrm{L}$ kanamycin and $100 \mathrm{mg} / \mathrm{L}$ streptomycin on a shaker at 180 $\mathrm{rpm}$ and at a temperature of $28^{\circ} \mathrm{C}$. The following day, $250 \mu \mathrm{l}$ of bacterial suspension was cultured into $10 \mathrm{ml}$ liquid LB medium at $28^{\circ} \mathrm{C}$ and $280 \mathrm{rpm}$ for 2 to $6 \mathrm{~h}$ until the desired density at $O D_{600} \mathrm{~nm}$ was obtained.

\section{Optimization of transient GUS expression parameters}

Several factors affecting the Agrobacterium-mediated transformation frequency in indica rice MR219 callus were evaluated. The factors were: Agrobacterium concentration (0.1, 0.2, 0.4, 0.6, 0.8 and 1.0 at $\left.\mathrm{OD}_{600} \mathrm{~nm}\right)$, age of embryogenic callus $(3,6,9$ and 12 weeks old), pre-culture period $(1,2,3,4,5,6$ and 7 days), wounding technique (no wounding, mild wounding-mash and massive wounding with scalpel), co-cultivation period (1, 2, 3, 4, 5 and 6 days), immersion time (30,60,90 and $120 \mathrm{~min})$, duration of dry time before co-cultivation $(15,30,45,60$ and $90 \mathrm{~min})$, acetosyringone concentration in immersion medium $(0,100,200$ and $300 \mu \mathrm{M})$, pH of co-cultivation media $(5.0,5.2,5.4,5.6,5.8$ and 6.0) and temperature of the co-cultivation period $\left(22,26\right.$ and $\left.28^{\circ} \mathrm{C}\right)$. To determine the optimum conditions for transformation, factor of the standard conditions were changed each time and the effects on percentage of transient gus $A$ gene expression were evaluated. At the end of the co-cultivation period, the embryogenic calli were evaluated and optimized on the basis of GUS expression (blue spots). Results obtained were based on the percentage of calluses expressing GUS spots over the total number of inoculated callus which was observed 3 days post-transformation. A sample was scored as GUS positive if the blue-spots on calluses mass were at least $25 \%$. All experiments were carried out with 50 embryogenic callus samples and repeated three times. 


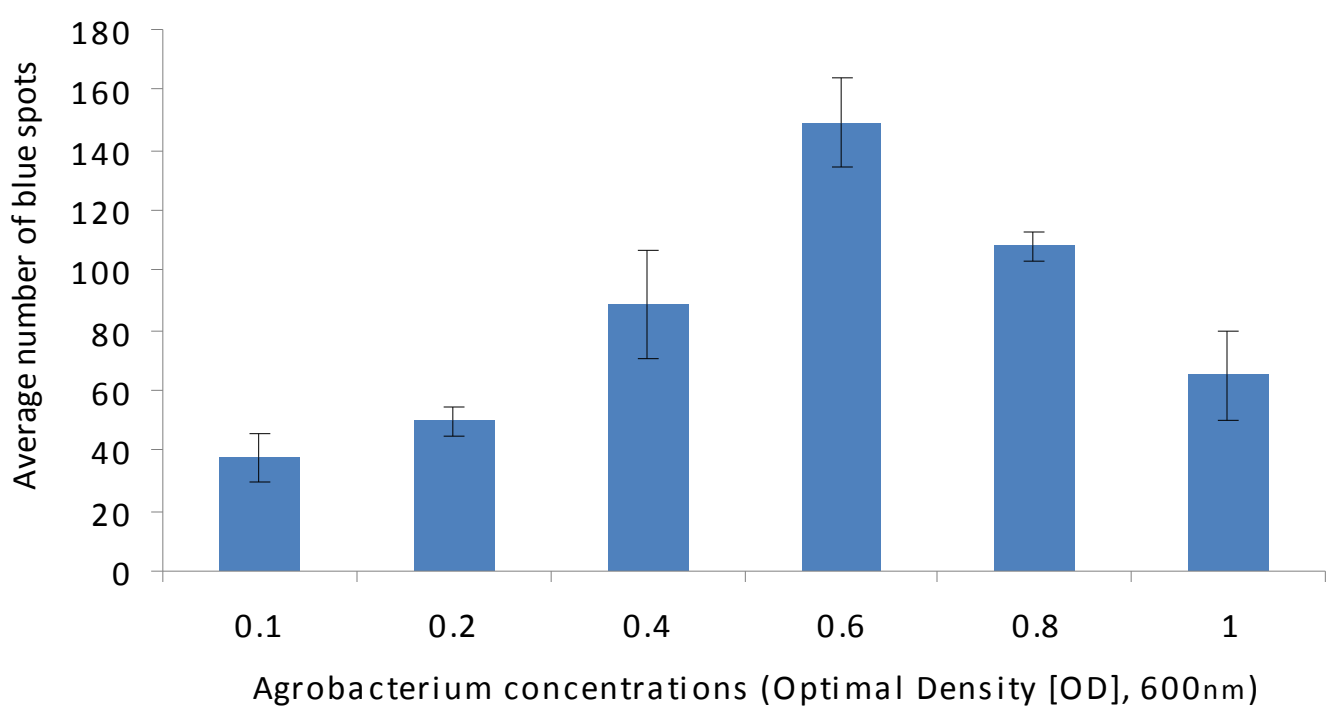

Figure 2. Effect of various Agrobacterium concentrations at $\mathrm{OD} 600_{\mathrm{nm}}$ on transient GUS expression in indica rice MR219 embryogenic callus. All experiments were carried out with 50 embryogenic callus samples and repeated three times.

\section{GUS histochemical assay}

The putative transformants callus was histochemically detected for the presence of the GUS gene following the procedure of Jefferson et al. (1987). The samples from the putative transgenic callus were incubated in GUS buffer [containing the stocks of $2 \mathrm{mM} \mathrm{X-}$ glucuronide in DMSO, $100 \mathrm{mM}$ Tris $\mathrm{HCl}(\mathrm{pH} 7.0), 50 \mathrm{mM} \mathrm{NaCl}, 2$ $\mathrm{mM}$ potassium ferricyanide and $0.1 \%(\mathrm{v} / \mathrm{v})$ triton $\mathrm{X}-100]$ overnight at $37^{\circ} \mathrm{C}$ and cleared in $75 \%$ ethanol for $4 \mathrm{~h}$ to clean the tissue prior observation. Transient GUS activity was recorded as blue spots (irrespective of size) using a stereomicroscope and photographed using a Nikon camera.

\section{Statistical analysis}

The analyses of variances were done and means were compared by the Duncan's multiple range test (DMRT) using SPSS program 9.0 (SPSS Inc. USA).

\section{RESULTS AND DISCUSSION}

The efficiency of Agrobacterium-mediated system on rice calli was inspected through vigilant observations on the effect of several parameters considered to be critical steps. The success of transformation was assessed by the percentage of blue spots signifying transient expression of GUS gene.

The density of Agrobacterium directly affects transformation efficiency since gene transfer only occurs with proper Agrobacterium attachment to plant cells. Therefore high Agrobacterium concentration increased the number of plant cells being infected (Sahoo et al., 2003). Figure 2 shows significant increase in the transformation rate with the increases of Agrobacterium concentration from 0.1 to 0.6 of $\mathrm{OD}_{600} \mathrm{~nm}$. The highest number of GUS staining was observed in Agrobacterium concentration $\mathrm{OD}_{600} \mathrm{~nm}$ at 0.6. More concentrated Agrobacterium suspension (when $\mathrm{OD}_{600} \mathrm{~nm}$ was 0.8-1.0) however, significantly reduced the number of transformed cells. This might be due to the fact that intense Agrobacterium infection caused severe damage to the plant cells, thus halting transformation process. Xing et al. (2007) reported that bacterium concentration at $\mathrm{OD}_{600} \mathrm{~nm}=0.6$ to 0.8 was the most efficient in sweet potato embryogenic callus Agrobacterium-mediated transformation. Karami (2008) reviewed that Agrobacterium concentration for transformation is dependent on multifactors including Agrobacterium strain and viability, plant species and cultivar and type of tissue used. Experiments with various rice genotypes and wheat showed that higher Agrobacterium density could increase transient GUS expression, but was not correlated with higher stable transformation frequency (Cheng et al., 1997; Hiei et al., 1997; Kumar et al., 2005; Nishimura et al., 2006).

The embryogenic callus of indica rice variety MR219 was selected as the target explants for transformations study. Hiei et al. (1997) demonstrated that embryogenic callus derived from mature seeds of japonica was the best target explants for Agrobacterium-mediated transformation due to its active cell division. Subsequently, freshly isolated immature embryos were later found to be the best explants type for indica rice (Aldemita and Hodges, 1996) and maize (Ishida et al., 2007). Bull et al. (2009) reviewed an overwhelming success of Agrobacterium-mediated transformation of friable embryogenic callus (FEC) in cassava; it is superior as a result of reduced risk of chimeric generation and reduced 


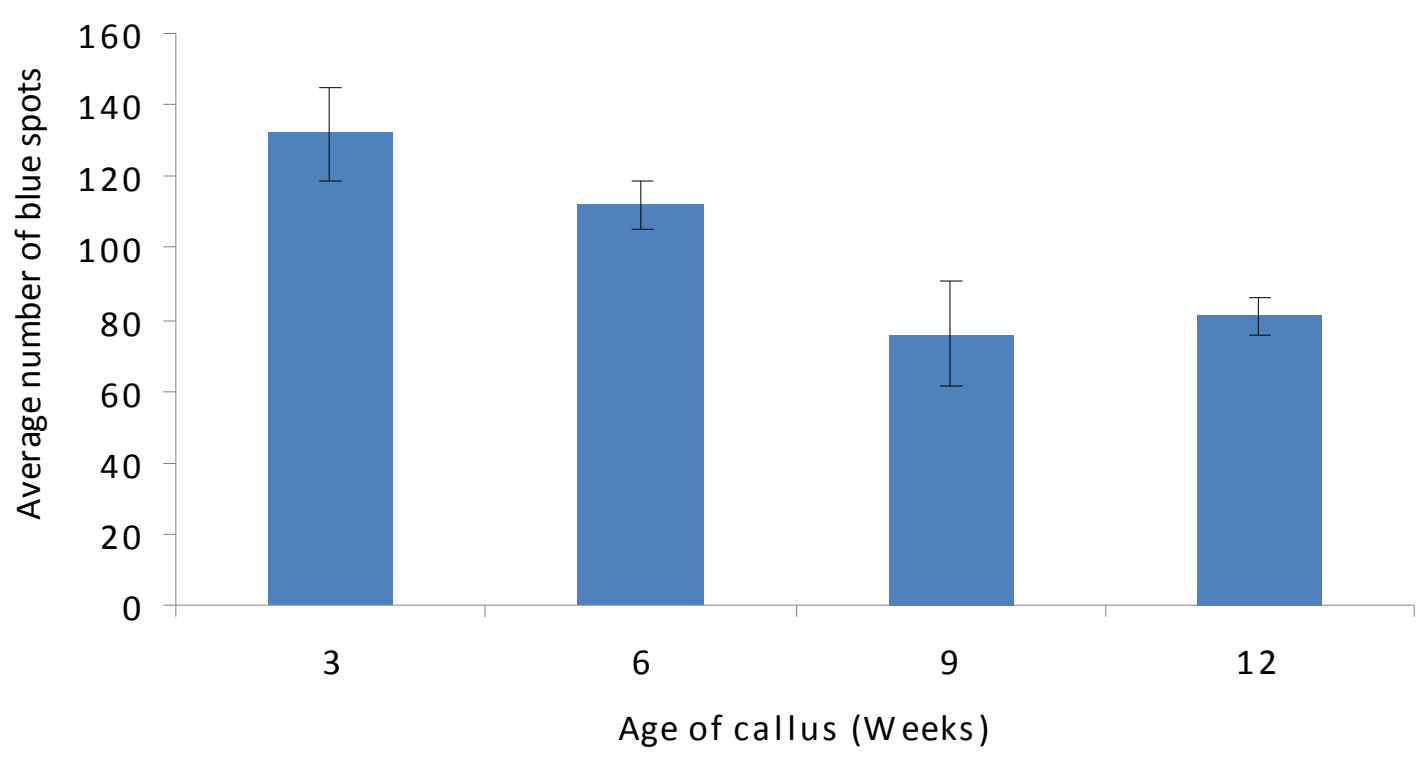

Figure 3. Effect of the age of callus on transient GUS expression in indica rice MR219 embryogenic callus. All experiments were carried out with 50 embryogenic callus samples and repeated three times.

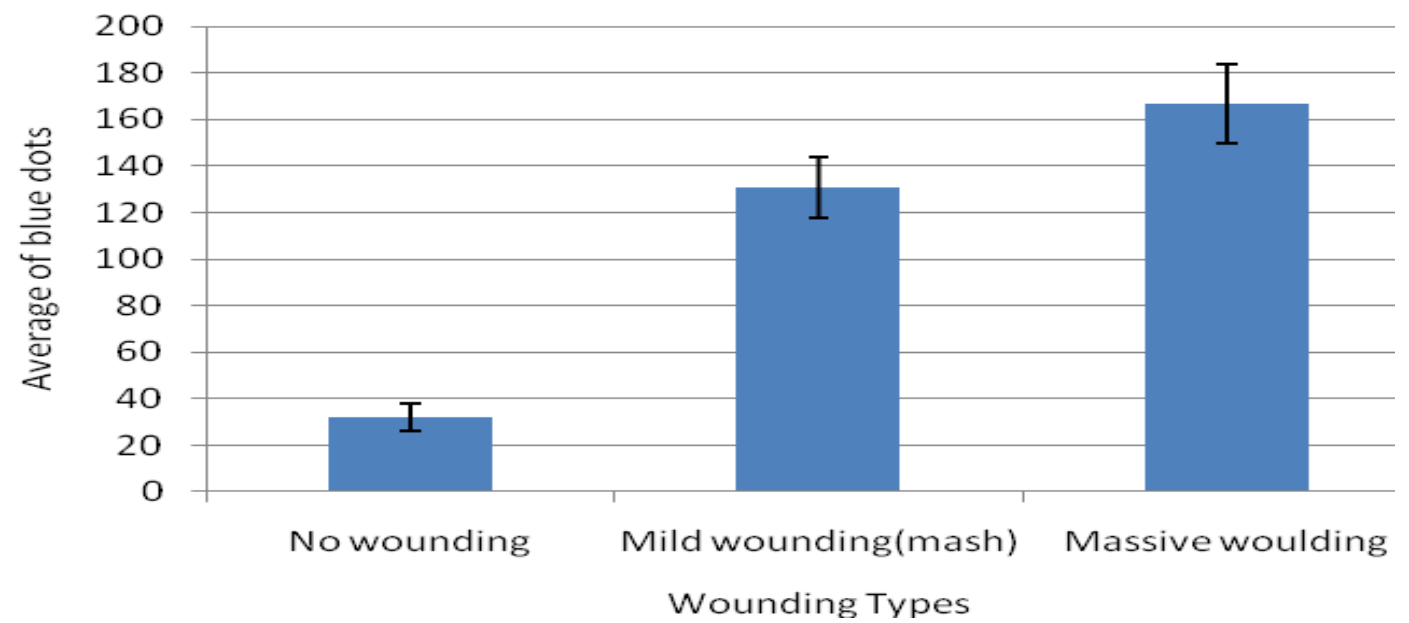

Figure 4. Effect of different wounding types of callus on transient GUS expression in indica rice MR219 embryogenic callus. All experiments were carried out with 50 embryogenic callus samples and repeated three times.

non-transformed plantlets escapes during selection as compared to organized tissues such as shoot organogenesis and cotyledon. The first efficient method for Agrobacterium-mediated transformation in japonica rice revealed the use of actively dividing, mature seeds derived embryogenic callus as suitable target explants (Nishimura et al., 2006).

The age of the callus is a crucial factor for transformation efficiency. Figure 3 illustrated the reduced transformation rate of embryogenic callus over time. Young calli of 3 weeks old highly enhanced transient GUS expressions as compared to older calli of 6 weeks old and above. Callus of more than 6 weeks old are more recalcitrant to Agrobacterium infection, due to increased immunity which is acquired by plant as defense to pathogen attack. Callus aged less than 3 weeks however, are too friable and fragile to endure bacterial infection (data not shown). Hiei and Komari (2008) reported that fresh and healthy immature embryos ensure successful japonica and indica rice transformation. Young embryogenic callus is also favourable due to its higher regeneration ability as compared to old calli (Raja et al., 2009).

Figure 4 shows the drastic increase (more than $70 \%$ ) of gene transfer in wounded plant as compared to intact 


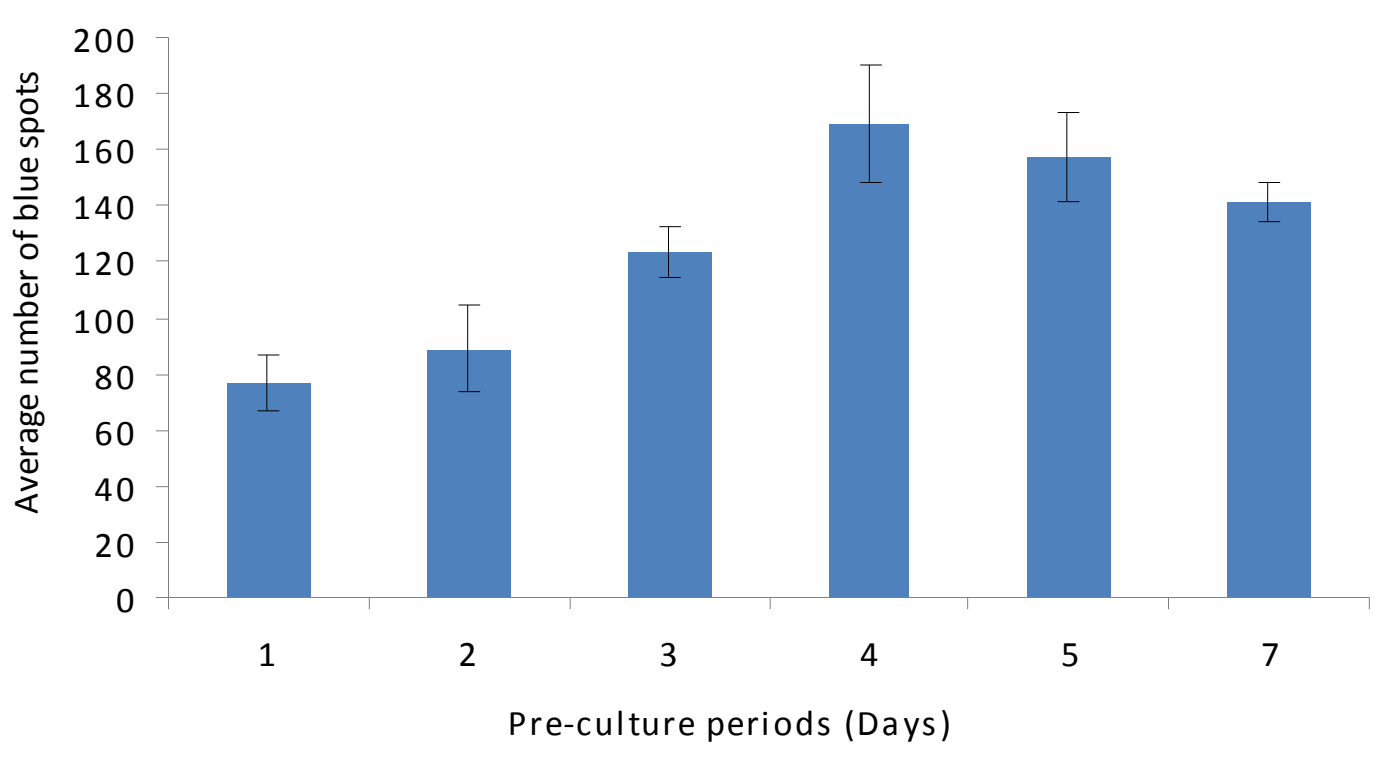

Figure 5. Effect of pre-culture periods on transient GUS expression in indica rice MR219 embryogenic callus. All experiments were carried out with 50 embryogenic callus samples and repeated three times.

explants. In nature, Agrobacterium infected wounded plant result to tumourigenesis which is known as crown gall disease. There are many types of wounding applied in plant transformation such as simple small incision using blades, micro-wounding using particle gun or sonication and injection by syringe (Sreeramanan et al., 2006). Karami (2008) reviewed that the idea of wounding the plant is to create an entry pathway for the Agrobacterium infection, in order to compromise physical barrier that might block T-DNA transfer, such as the waxy cuticles on plant epidermis; to stimulate phenolic compound necessary for vir gene activation release; and that wounding makes plant cell to actively divide and makes cell walls to be less rigid for easy bacterium breaching, and active DNA replication at this point might facilitate T-DNA integration.

Shrawat et al. (2007) defined pre-culture period as the period that starts the moment immature embryo are first isolated and cultured until immediately before Agrobacterium infection. Figure 5 demonstrates that extending pre-culture period more than 3 days improved transformation frequency. Four (4) days of pre-culture proved to be the best increasing transformation efficiency by nearly $50 \%$ as compared to only 1 day pre-culture. Longer pre-culture time ( 5 and 6 days) although enhanced transformation, the efficiency however reduced after the 4th day of pre-culture. Extended pre-culture period allow embryogenic calli to maximize nutrient uptake from pre-culture media to stimulate cells to become competent for Agrobacterium attachment. In the transformation of Vigna sp., Sahoo et al. (2003) stated that during pre-culture, a physiological and developmental shift occurred in plant tissues to become regenerate-competent which consequently lead to the success of transformation.

Co-cultivation period is described as the time between the elimination of unattached Agrobacterium after inoculation, and when the calli are subjected to observation or selection of transgenic line. At this stage, embryogenic calli are cultured together with attached Agrobacterium for further infection process on medium lacking selection pressure and anti Agrobacterium component such as cefotaxime. Co-cultivation period of embryogenic callus also exhibit the same pattern to the pre-culture period (Figure 6). Significant increased of transient GUS expression were observed in 4 days of co-cultured calli. Lesser days ( 1 to 3 days) of co-cultivation period produced few transformed line due to insufficient time for complete or maximized transfer of Agrobacterium T-DNA into the plant genome. Extended time (5 and 6 days) of co-cultivation also significantly reduced trans-formation due to overgrown bacteria damage and suffo-cation of the explants. Huang and Wei (2005) reported that no transformation occurred in immature embryo of maize without co-cultivation period. They also stated that prolonged co-cultivation period reduced transformed immature embryo frequency due to abundant proliferation of bacterium.

The effect of infection period against drying period after infection was carried out and data are summarized in Table 1. In general, 30 min of drying period regardless of infection period was optimal for rice calli transformation in this study whereas, $90 \mathrm{~min}$ is the optimal infection period to ensure high transformation efficiency. The highest number of GUS activity was observed in calli infected for $90 \mathrm{~min}_{2}$ followed by $30 \mathrm{~min}$ of drying on sterile filter paper 


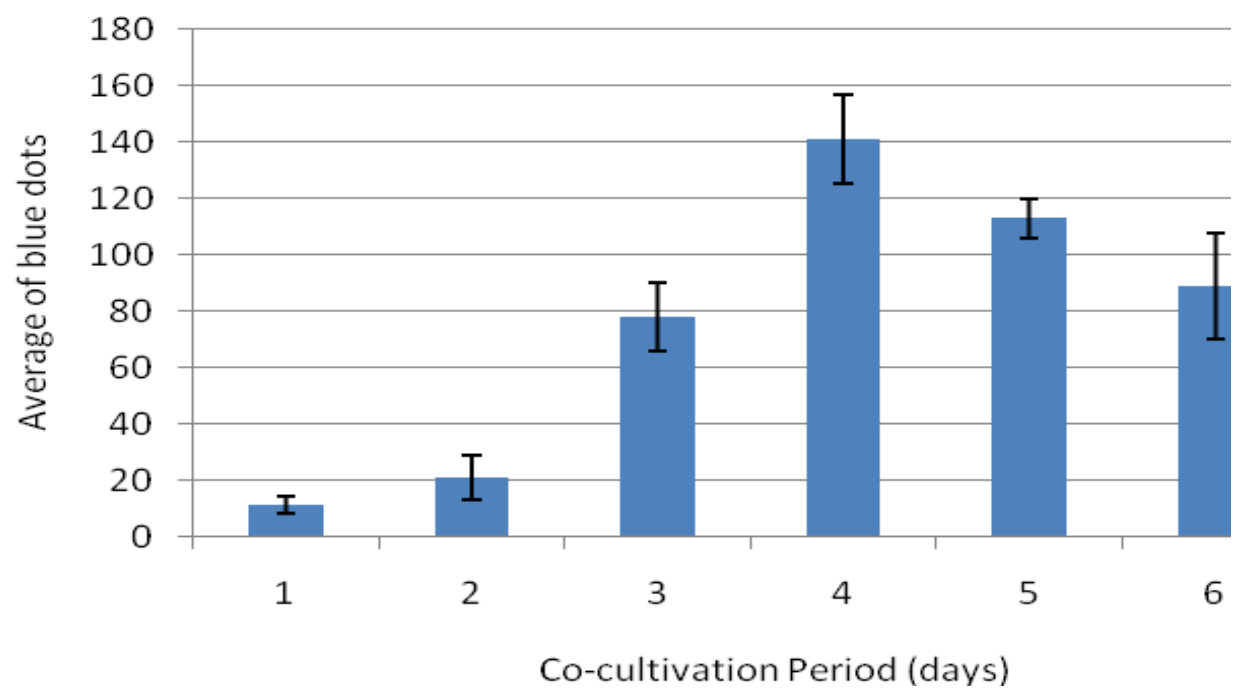

Figure 6. Effect of co-culture periods on transient GUS expression in indica rice MR219 embryogenic callus. All experiments were carried out with 50 embryogenic callus samples and repeated three times.

Table 1. Effect of various drying periods after infection on indica rice MR219 embryogenic callus. All experiments were carried out with 50 embryogenic callus samples and repeated three times.

\begin{tabular}{llllll}
\hline \multirow{2}{*}{ Infection period ( $\mathbf{m i n})$} & \multicolumn{5}{c}{ Average of } \\
\cline { 2 - 6 } & $\mathbf{1 5}$ & $\mathbf{3 0}$ & $\mathbf{4 5}$ & $\mathbf{6 0}$ & $\mathbf{9 0}$ \\
\hline 30 & $51 \pm 7$ & $57 \pm 5$ & $46 \pm 5$ & $26 \pm 8$ & $34 \pm 5$ \\
60 & $63 \pm 5$ & $74 \pm 12$ & $64 \pm 6$ & $49 \pm 4$ & $57 \pm 12$ \\
90 & $71 \pm 7$ & $167 \pm 16$ & $154 \pm 17$ & $63 \pm 12$ & $66 \pm 6$ \\
120 & $101 \pm 17$ & $123 \pm 10$ & $46 \pm 8$ & $56 \pm 6$ & $41 \pm 7$ \\
\hline
\end{tabular}

prior to co-cultivation. The combination produce transformation rate recorded almost $85 \%$ higher than the combination of $30 \mathrm{~min}$ of infection and 60 min drying period which only recorded $26 \pm 8$ of the average blue dots.

The prolonged immersion period of $90 \mathrm{~min}$ allowed sufficient time for a large number of Agrobacterium to efficiently get attached to plant cells for infection process, and eventually highly influenced the transformation in calli. Drying period of $30 \mathrm{~min}$ is very suitable in MR219 embryogenic calli transformation of which the period is adequate to eliminate unattached bacteria from the explants and to preserve the cells freshness from losses of water content. Desiccation of precultured immature embryo in wheat after Agrobacterium infection was shown to lead to higher efficient transformation rate (Cheng et al., 2003). However, freshly isolated immature embryos and precultured immature embryos of sorghum showed higher competency in Agrobacterium infection as compared to those that had undergone desiccation process (Zhao et al., 2000).

Figure 7 illustrates the influence of various acetosyringone concentrations $(0,100,200$ and $300 \mu \mathrm{M})$ in the immersion medium on the transformation of embryogenic callus. Significant high GUS staining was achieved at the addition of $200 \mu \mathrm{M}$ acetosyringone in the immersion medium. Similar result was obtained in the transformation of sweet potato when the effect of acetosyringone concentration in co-cultivation medium was investigated (Xing et al., 2007). Acetosyringone is a type of phenolic compound which is well known to increase Agrobacterium vir gene activity. However, like most monocot, rice is unable to synthesize such compound. Successful transformation record in barley proved that acetosyringone is not an essential factor; however inclusions of the compound in co-culture medium significantly enhanced gus or gfp expression in barley embryo (Shrawat et al., 2007) and Phalaenopsis violacea orchid (Sreeramanan and Xavier, 2010). They also stated that inclusion of acetosyringone is important when GUS activity is used to determine transformed embryo; whereas, successful gene delivery into embryo can still be observed based on gfp expression if acetosyringone is neglected. 


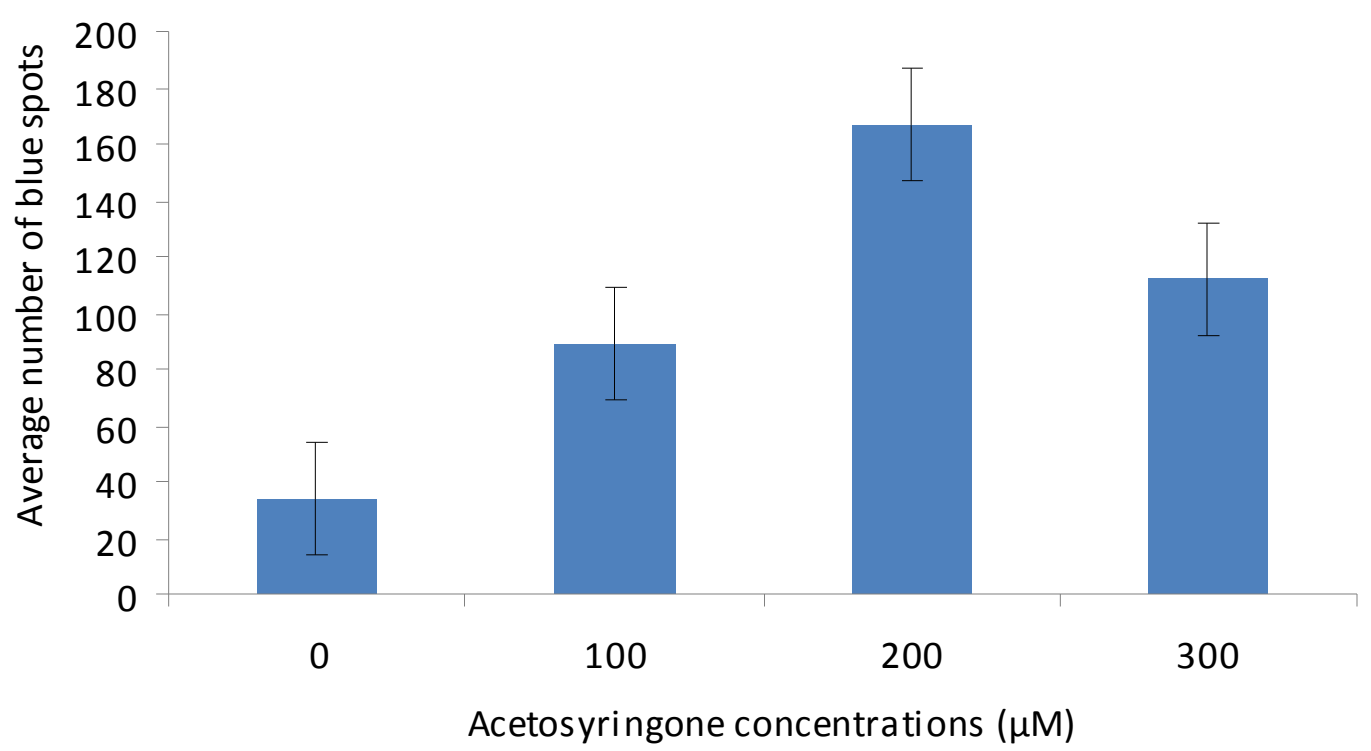

Figure 7. Effect of acetosyringone concentrations on transient GUS expression in indica rice MR219 embryogenic callus. All experiments were carried out with 50 embryogenic callus samples and repeated three times.

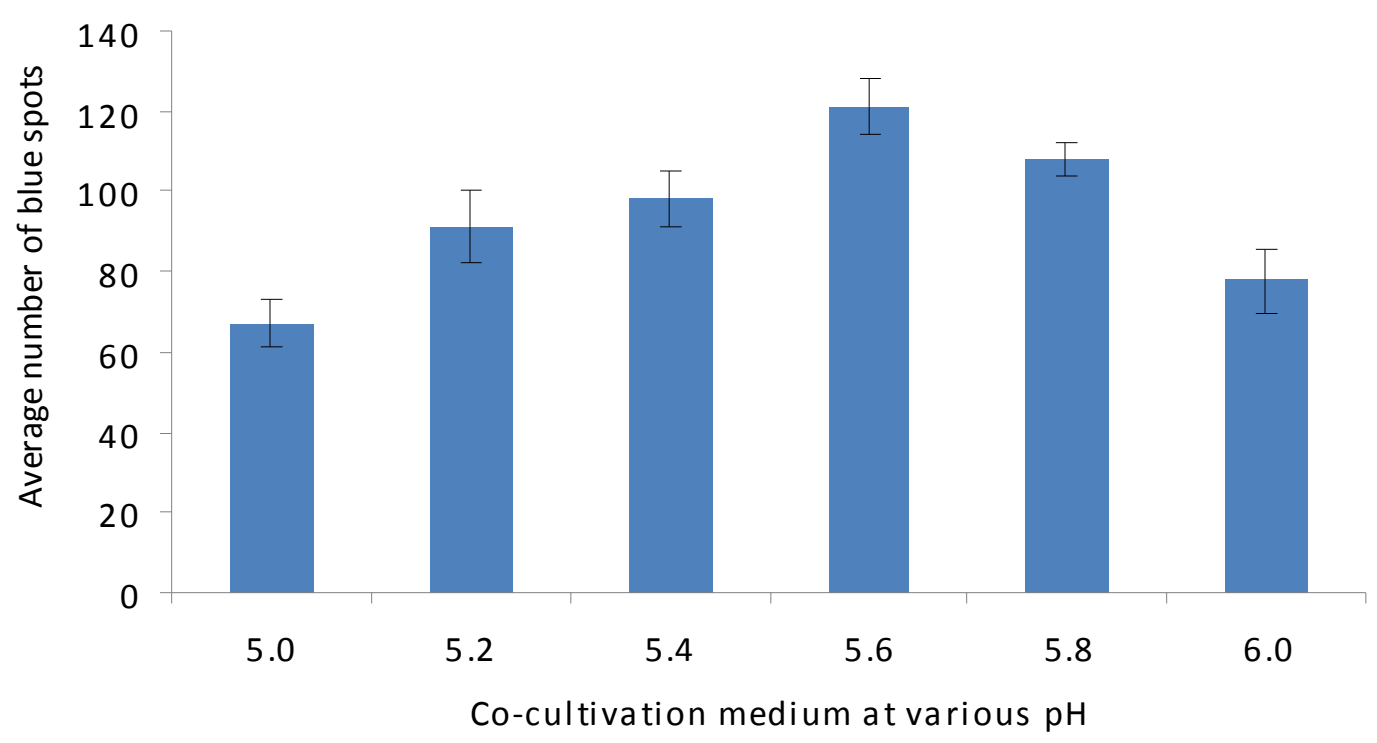

Figure 8. Effect of various $\mathrm{pH}$ of co-culture medium on transient GUS expression in indica rice MR219 embryogenic callus. All experiments were carried out with 50 embryogenic callus samples and repeated three times.

Increasing GUS activity was observed in increasing $\mathrm{pH}$ of co-cultivation medium until pH 5.6 (Figure 8). The highest detection of GUS expression of the transformed explants was detected in $\mathrm{pH} 5.6$ followed by $\mathrm{pH} 5.8$. However, at $\mathrm{pH} 5.4$, we could detect 98 blue spots as compared to at $\mathrm{pH} 5.2$ where only 92 blue spots were produced, respectively (Figure 8 ). The $\mathrm{pH}$ of the cocultivation period was found to be equally an important factor assisting the transformation efficiency. The highest gfp expressions of PLBs were observed in $\mathrm{pH} 5.5$ which is a slightly acidic condition (Sreeramanan and Zuraida, 2010). Huang and Wei (2005) obtained the highest PPTresistance maize calli at slight acidic $\mathrm{pH}$ of 5.4 . It has been suggested that the acidic $\mathrm{pH}$ of 5.5 is suitable for the transformation process as it may induce the virulence genes required for gene transfers (Stachel et al., 1986; 


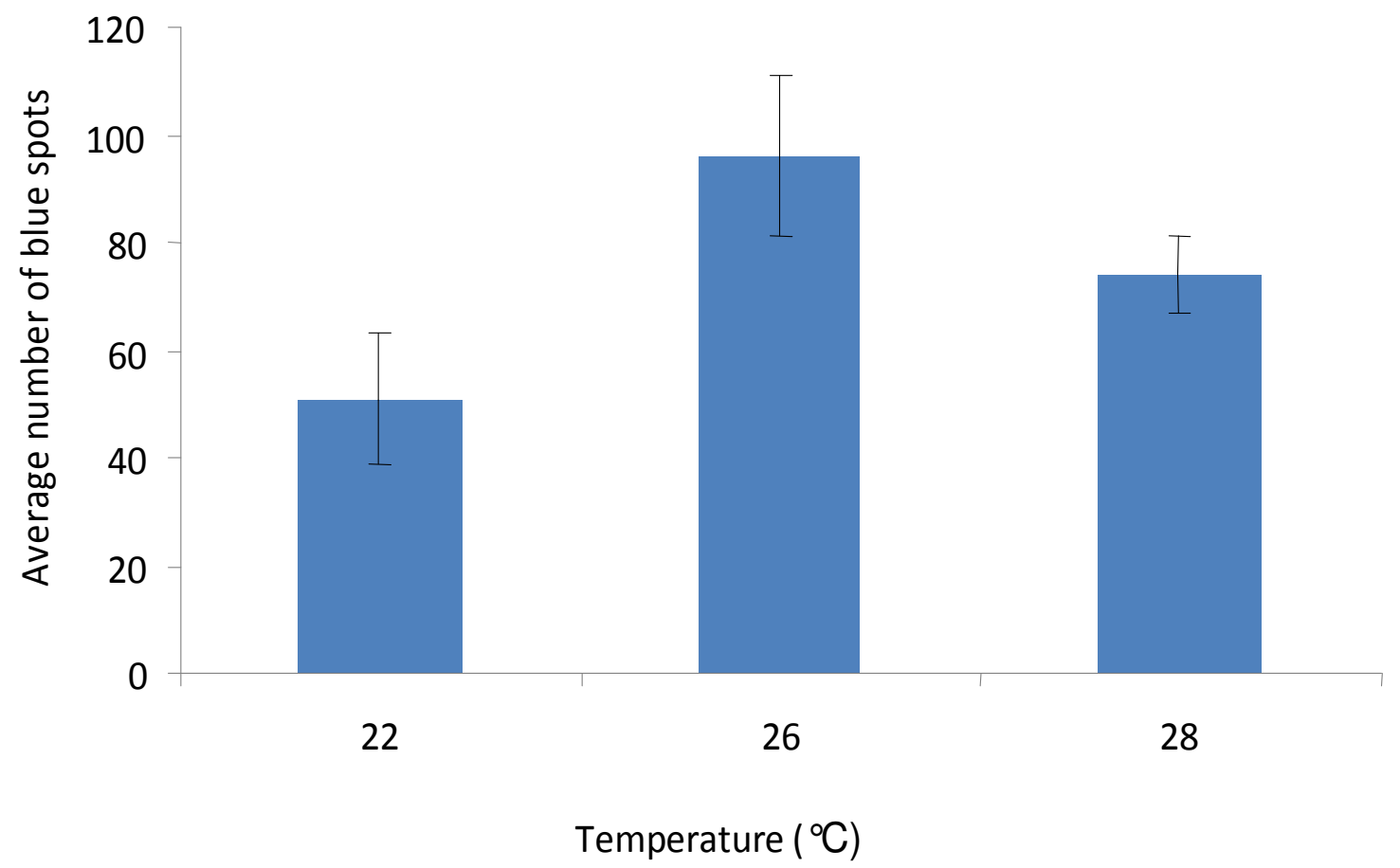

Figure 9. Effect of different temperatures during co-cultivation period on transient GUS expression in indica rice MR219 embryogenic callus. All experiments were carried out with 50 embryogenic callus samples and repeated three times.

Huang and Wei, 2005). The acidic pH of co-cultivation medium suitable for the Agrobacterium-mediated transformation process is due to its possible function in the induction of vir genes and initiation of T-DNA transfer (Huang and Wei, 2005).

Temperature has been considered a factor affecting the capacity of Agrobacterium to transfer the T-DNA to plant cells (Karami, 2008; Sreeramanan and Xavier, 2010). The investigation of the effect of temperature during cocultivation in MR219 embryogenic calli revealed that temperature plays an important role in transformation efficiency. Higher temperature of $26^{\circ} \mathrm{C}$ was found to be optimal to support the highest transient transformation frequency in this rice cultivar and there was no difference between 26 and $28^{\circ} \mathrm{C}$ (Figure 9). Dramatic transient expression reduction occurred when temperature decreased from 26 to $22^{\circ} \mathrm{C}$. The results are contradictory to various studies in which optimal temperatures for transformation ranged from 19 to $25^{\circ} \mathrm{C}$. However, due to high inconsistency across plant species and tissue used, it is possible to affirm that the optimal temperatures varied depending on these factors.

Therefore, an efficient Agrobacterium-mediated transformation protocol for indica rice MR219 was successfully established at this stage. The investigation of various factors that influence T-DNA delivery is an important first step in the utilization of Agrobacterium in the transformation of indica rice MR219 calli.

\section{REFERENCES}

Aldemita RR, Hodges TK (1996). Agrobacterium tumefaciens-mediated transformation of japonica and indica rice varieties. Planta, 199: 612617.

Bull SE, Owiti JA, Niklaus M, Beeching JR, Gruissem W, Vanderschuren H (2009). Agrobacterium-mediated transformation of friable embryogenic calli and regeneration of transgenic cassava. Nat. Protoc. 4(12): 1845-1854.

Cheng M, Fry JE, Pang S, Zhou H, Hironaka C, Duncan DR, Conner TW, Wan Y (1997). Genetic transformation of wheat mediated by Agrobacterium tumefaciens. Plant Physiol. 115: 971-980.

Cheng M, Hu T, Layton J, Liu CN, Fry JE (2003). Desiccation of plant tissues post-Agrobacterium infection enhances T-DNA delivery and increases stable transformation efficiency in wheat. In Vitro Cell. Dev. Biol. Plant. 39(6): 595-604.

Hiei Y, Komari T (2008). Agrobacterium-mediated transformation of rice using immature embryos or calli induced from mature seed. Nat. Protoc. 3(5): 854-834.

Hiei Y, Komari T, Kubo T (1997). Transformation of rice mediated by Agrobacterium tumefaciens. Plant Mol. Biol. 35: 205-218.

Huang X, Wei Z (2005). Successful Agrobacterium-mediated genetic transformation of maize elite inbred lines. Plant Cell. Tissue Org. Cult. 83: 187-200.

Hui CE, Ping Z, Min ZS, H LA, Fang ZY, Xiang CZ, Biao PX (2004). Factors affecting Agrobacterium-mediated transformation efficiency in rice. Rice Sci. 11(4): 181-185.

Ishida Y, Hiei Y, Komari T (2007). Agrobacterium-mediated transformation of maize. Nat. Protoc. 2(7): 1614-1621.

Jefferson RA (1987). Assaying chimeric gene in plants: the GUS gene fusion system. Plant Mol. Biol. Rep. 5: 387-405.

Jiao Y, Wang Y, Xue D, Wang J, Yan M, Liu G, Dong G, Zeng D, Lu Z, Zhu X, Qian Q, Li J (2010). Regulation of OsSPL14 by OsmiR156 defines ideal plant architecture in rice. Nat. Gen. 42(6): 541-545.

Karami O (2008). Factors affecting Agrobacterium-mediated trans- 
formation of plants. Trans. Plant J. 2(2): 127-137.

Kavitha G, Taghipour F, Huyop F (2010). Investigation of factors in optimizing Agrobacterium-mediated gene transfer in Citrullus lanatus cv. Round Dragon. J. Biol. Sci. 10(3): 209-216.

Kumar KK, Maruthasalam S, Loganthan M, Sudhakar D, Balasubramaniam P (2005). An improved Agrobacterium-mediated transformation protocol for recalcitrant elite indica rice cultivars. Plant Mol. Bio. Rep. 23: 6-73.

Miura K, Ikeda M, Matsubara A, Song X-J, Ito M, Asano K, Matsuoka M, Kitano H, Ashikari M (2010). OsSPL14 promotes panicle branching and higher grain productivity in rice. Nat. Gen. 42(6): 545-549.

Murashige T, Skoog F (1962). A Revised Medium For Rapid Growth and Bioassay with Tobacco Tissue Culture. Plant Physiol. 15: 473497.

Nishimura A, Aichi I, Matsuoka M (2006). A protocol for Agrobacteriummediated transformation in rice. Nat. Protoc. 1(6): 2796-2802.

Raja NI, Bano A, Rashid H, Khan MH, Chaudhry Z (2009). Effect of age of embryogenic callus on plant regeneration in local cultivars of wheat (Triticum aestivum L.). Pak. J. Bot. 41(6): 2801-2806.

Sahoo L, Sugla T, Jaiwal PK (2003). In-vitro regeneration and genetic transformation of Cowpea, Mungbeam Urdbean and Azuki bean. Appl. Gene. Leguminose Biotechnol. Ed. Jaiwal PK and Singh RP. Kluwer Academic Publisher, Great Britain. pp. 89-120.

Shrawat AK, Becker D, Lo rz H (2007). Agrobacterium tumefaciensmediated genetic transformation of barley (Hordeum vulgare L.). Plant Sci. 172: 281-290.

Sreeramanan S, Maziah M, Abdullah MP, Sariah M, Xavier R (2006). Transient expression of gusA and gfp gene in Agrobacteriummediated banana transformation using tiny meristematic bud. Asian J. Plant Sci. 5: 468-480.
Sreeramanan S, Xavier R (2010). Emerging factors that influence efficiency of T-DNA gene transfer into Phalaenopsis violacea orchid via Agrobacterium tumefaciens-mediated system. Int. J. Biol. 2(2): 64-73

Sreeramanan S, Zuraida AR (2010). Early GFP gene assessments influecing Agrobacterium tumefaciens transformation system in Phalaenopsis violacea orchid. Emir. J. Food Agric. 22(2): 103-116.

Stachel SE, Nester, EW and Zambryski, PC (1986). A plant cell factor induces Agrobacterium tumefaciens vir gene expression. Proc. Natl. Acad. Sci. USA, 83: 379-383.

Xing Y, Yang Q, Ji Q, Luo Y, Chang Y, Gi K, Wang D (2007). Optimization of Agrobacterium-mediated transformation parameters of sweet potato embryogenic callus using $\beta$-glucuronidase (GUS) as a reporter. Afr. J. Biotechnol. 6(22): 2578-2584.

Zhao ZY, CAi T, Tagliani L, Miller M, Wang N, Pang H, Rudert M, Schroeder S, Hondred D, Seltzer J, Pierce D (2000). Agrobacteriummediated sorghum transformation. Plant Mol. Biol. 44: 789-798.

Zuraida AR, Hamidah G, Habibuddin H, Shaiful AS, Marzukhi H, Naziah $B$, Zulkifli AS (2007). Optimization of callus induction for Malaysian indica rice variety MR219. Proceeding of USM-UPM- PETA Conference 2007, Evergreen Laurel Hotel, Penang. 28-29 November 2007. 